\title{
Intraoperative blood loss in oncological spine surgery
}

\author{
*Malte Mohme, MD, ${ }^{1}$ Klaus C. Mende, MD, ${ }^{1}$ Tobias Pantel, MD, ${ }^{1}$ Lennart Viezens, MD, ${ }^{2}$ \\ Manfred Westphal, MD, ${ }^{1}$ Sven Oliver Eicker, MD, ${ }^{1}$ Marc Dreimann, MD, ${ }^{2}$ Theresa Krätzig, MD, ${ }^{1}$ and \\ Martin Stangenberg, MD²
}

\begin{abstract}
1Department of Neurosurgery and 2Division of Spine Surgery, Department of Trauma and Orthopedic Surgery, University Medical Center Hamburg-Eppendorf, Hamburg, Germany
\end{abstract}

OBJECTIVE Intraoperative blood loss in patients undergoing oncological spine surgery poses a major challenge for vulnerable patients. The goal of this study was to assess how the surgical procedure, tumor type, and tumor anatomy, as well as anesthesiological parameters, affect intraoperative blood loss in oncological spine surgery and to use this information to generate a short preoperative checklist for spine surgeons and anesthesiologists to identify patients at risk for increased intraoperative blood loss.

METHODS The authors performed a retrospective analysis of 430 oncological patients who underwent spine surgery between 2013 and 2018 at the university medical spine center. Enrolled patients had metastatic tumor of the spine requiring surgical decompression of neural structures and/or stabilization including tumor biopsy using an open, percutaneous, and/or combined dorsoventral approach. Patients requiring vertebro- and kyphoplasty or biopsy only were excluded. Statistical analyses performed included a multiple linear regression analysis.

RESULTS The mean intraoperative blood loss in the study patient cohort was $1176 \pm 1209 \mathrm{ml}$. In total, $33.8 \%$ of patients received intraoperative red blood cell transfusions. The statistical analyses showed that tumor histology indicating myeloma, operative procedure length, epidural spinal cord compression (ESCC) score, tumor localization, BMI, and surgical strategy were significantly associated with increased intraoperative blood loss or risk of needing allogeneic blood transfusions. Anesthesiological parameters such as the American Society of Anesthesiologists (ASA) Physical Status classification score were not associated with blood loss. Multiple linear regression analysis demonstrated good predictive value ( $r$ $=0.437$ ) for a five-item preoperative checklist to identify patients at risk for high intraoperative blood loss.

CONCLUSIONS The analyses performed in this study demonstrated key factors affecting intraoperative blood loss and showed that a simple preoperative checklist including these factors can be used to identify patients undergoing surgery for metastatic spine tumors who are at risk for increased intraoperative blood loss.

https://thejns.org/doi/abs/10.3171/2021.2.FOCUS201117

KEYWORDS spine tumor; tumor decompression; blood loss; transfusion; oncological spine surgery

W ITH advances in cancer therapy and the resulting increase in life expectancy of patients in the metastatic disease stage, the incidence and thus the need for treatment of spinal metastases, with the main goals of preserving or regaining neurological function and providing pain relief, continue to rise. ${ }^{1-3}$ In recent years, with the various scoring systems developed to support the practitioner in making therapeutic decisions ${ }^{3-6}$ and significant improvements in surgical techniques and radiosurgery modalities, spinal metastases can be treated with durable local tumor control. ${ }^{7,8}$ Minimally invasive techniques have been shown to significantly reduce not only the length of hospitalization, but also the perioperative morbidity and intraoperative blood loss. ${ }^{9-11}$ Because the surgical treatment of both spinal and extraspinal metastases is often associated with considerable blood loss (mean $1200 \mathrm{ml}$, up to $6000 \mathrm{ml}$ ), intraoperative and perioperative bleeding management has increased significantly in importance and became a pivotal topic,,$^{12}$ especially when minimally invasive techniques alone are not sufficient to regain, e.g., spinal alignment due to vertebral collapse and hyperkyphosis.

Numerous factors, such as tumor histology, type of surgical procedure, and duration of surgery, are known to influence the amount of intraoperative blood loss. ${ }^{13}$ However, anesthesiology parameters, percentage of osseous

ABBREVIATIONS ABT = allogeneic blood transfusion; $\mathrm{ASA}=$ American Society of Anesthesiologists; ESCC = epidural spinal cord compression; KW = Kruskal-Wallis; MET $=$ metabolic equivalent of task; $\mathrm{RBC}=$ red blood cell.

SUBMITTED December 31, 2020. ACCEPTED February 17, 2021.

INCLUDE WHEN CITING DOI: 10.3171/2021.2.FOCUS201117.

* T.K. and M.S. contributed equally to this work. 
involvement, epidural epidural spinal cord compression (ESCC) score,${ }^{14}$ and other factors that have not yet been investigated may also be important. The aim of our study was to identify factors that have a decisive influence on intraoperative blood loss in metastatic spine tumor surgery and to generate a preoperative checklist as a prediction model of the amount of intraoperative blood loss. Better anticipation of what to expect with intraoperative and perioperative bleeding in patients undergoing oncological spine surgery could lead to more appropriate preoperative planning, ideally optimizing the use of antifibrinolytic agents or embolization and helping to reduce the need for blood products.

\section{Methods \\ Patient Data}

We performed retrospective analyses on data sets of 430 patients with tumors of the spinal column treated at our institution from January 2013 to June 2018. We included all patients with metastatic tumor to the spine requiring surgical decompression of neural structures and/or stabilization including tumor biopsy using an open, percutaneous, and/or a combined dorsoventral approach. Patients requiring vertebro- and kyphoplasty or biopsy only were excluded. Data analysis was performed retrospectively on anonymized clinical data sets. The study was conducted in accordance with the guidelines of the local ethical review board (WF-042/18) and the Declaration of Helsinki.

\section{Data Acquisition}

Data were collected from electronic case records that included anesthesiological protocols. Data for the following parameters were extracted: age, sex, histology, presence of peripheral metastases, localization of metastases along the spine, numbers of vertebrae involved, instability as defined by Spine Instability Neoplastic Score (SINS) criteria, presence of fractures, type of bony affection, proportion of osseous involvement of tumor mass, procedures, American Society of Anesthesiologists (ASA) Physical Status classification score, metabolic equivalent of task (MET), BMI, estimated intraoperative blood loss, blood transfusions, crystalloids and colloid transfusions, length of surgery, and postoperative monitoring.

\section{Statistical Analysis}

Statistical analysis was performed using chi-square tests for nominal variables and the Student $t$-test for parametric measures. Nonparametric variables were analyzed using the Kruskal-Wallis (KW) test (with Bonferroni correction in the case of multiple comparisons) and MannWhitney U-test with Microsoft Excel 2019 and IBM SPSS version 26 (IBM Corp). Significance was defined with a $95 \%$ confidence interval, and $\mathrm{p}$ values $<0.05$ were considered significant. Graphical analysis was performed using GraphPad Prism 8.

\section{Results \\ Patient Cohort}

Overall, we included 430 oncological patients with extradural tumor manifestation who underwent surgical decompression and/or stabilization due to ESSC or tumor-associated instability during the time period from January 2013 to July 2018. In total, 62.6\% $(\mathrm{n}=269)$ of patients were male and $37.4 \%(\mathrm{n}=161)$ were female. The mean age was $64.1 \pm 0.585$ years, with a range of 23-91 years. Additional study population characteristics, including BMI $\left(\mathrm{kg} / \mathrm{m}^{2}\right)$, ASA Physical Status scores, and MET classifications are summarized in Table 1 . The mean intraoperative blood loss in our cohort was $1176 \pm 1209 \mathrm{ml}$. In total, $33.8 \%$ of patients received intraoperative red blood cell (RBC) transfusion and 4\% of patients received thrombocyte transfusions (Table 2). When patients received a transfusion, on average 2.75 units of RBCs and 1.29 units of thrombocytes were transfused. Intraoperatively administered crystalloid volumes were $2314 \pm 1099 \mathrm{ml}$ and colloid volumes were $418 \pm 629 \mathrm{ml}$ (Table 2).

\section{Histology}

Metastatic or primary tumors leading to ESSC or tumor-associated instability most commonly included prostate $(\mathrm{n}=69,16.0 \%)$, lung $(\mathrm{n}=65,15.1 \%)$, and breast $(\mathrm{n}=$ $64,14.9 \%)$ cancer; myeloma $(\mathrm{n}=50,11.6 \%)$; and renal $(\mathrm{n}=$ $36,8.4 \%)$ and colon $(\mathrm{n}=22,5.1 \%)$ cancer (Table 1). Additional metastatic entities and/or tumors are listed in Table 1. The group "other" included the following entities: adrenal cancer, cholangiocellular carcinoma, chordoma (only primary tumor), lymphoma, thyroid cancer, and germ cell tumors. The distribution of metastases along the spine, i.e., cervical (C0-T1), thoracic (T2-L1), lumbar (L2-S1), and sacral (S2-4), is shown in Fig. 1. Breast and gastric cancer showed a higher prevalence of cervical involvement compared with the other entities $(\mathrm{p}=0.028)$, while the frequency of colon cancer metastases was higher in the lumbar and sacral region of the spine $(\mathrm{p}=0.028)$ (Fig. 1). We found increased intraoperative blood loss in patients with myeloma $(2061 \pm 1797 \mathrm{ml})$, which was significant in pairwise comparison versus metastasis from breast and lung cancer, respectively $(\mathrm{p}=0.002$ and $\mathrm{p}<0.001, \mathrm{KW}$ test with Bonferroni correction for multiple comparisons) (Fig. 2). Below-average volumes of intraoperative blood loss were found in patients with lung cancer $(784 \pm 738$ $\mathrm{ml})$ and ureteral cancer $(657 \pm 469 \mathrm{ml})$ (Table 2, Fig. 2). Myeloma patients on average received 1.68 units of RBCs. In our cohort, only 6 patients underwent preoperative embolization (5 patients with renal cancer, 1 with thyroid carcinoma). Thus, the statistical impact of preoperative embolization was negligible in this cohort.

\section{Anatomy}

We additionally analyzed the amount of intraoperative blood loss according to the number of vertebrae affected (Fig. 2B). While there was a trend of increased blood loss when we compared 1 versus 3 involved vertebrae ( $\mathrm{p}$ $=0.056$, nonparametric t-test), no significant association could be demonstrated in the KW statistics throughout this parameter. When grouping patients according to the ESCC scale, we found a significant increase in intraoperative blood loss with increasing ESCC score $(p=0.035$, KW test) (Fig. 2C). Patients with an ESCC score of 1 (1a, 
$1 \mathrm{~b}$, or 1c) lost $890 \pm 739 \mathrm{ml}$ blood on average compared with $1291 \pm 1318 \mathrm{ml}$ blood loss in patients with an ESCC score of 3 ( $p=0.0186$, nonparametric t-test). An additional detailed assessment of the osseous involvement, determined as the percentage of tumor that involves the bone, showed no significant association with intraoperative blood loss (Fig. 2D). Analysis of the tumor location along the spinal axis, i.e., cervical, thoracic, lumbar, or sacral, demonstrated that patients with tumors of the sacral region (S2-4) in particular were at higher risk for needing large $\mathrm{RBC}$ transfusion amounts $(40 \%$ requiring $>3$ units; $\mathrm{p}=0.004, \mathrm{KW}$ test) than patients with tumor locations at the other regions: $\mathrm{RBC}>3$ units required in $6.9 \%$ of patients with cervical tumor location, $10.8 \%$ with thoracic, and $3.1 \%$ with lumbar (Fig. 2E).

\section{Anesthesia}

As oncological patients with metastases of the spine often display multiple comorbidities and represent a fragile patient population, we analyzed anesthesiological parameters. As expected, most patients included in our analysis had ASA scores of 3 (severe disease) or 4 (severe incapacitating disease with constant threat to life). In particular, patients with hepatocellular carcinoma and ureteral carcinoma showed a restricted general condition, with ASA scores of 3 or 4 in $100 \%$ of patients (Fig. 3A). On the other hand, in myeloma patients, who showed the highest intraoperative blood loss among all tumor entities, only 55.3\% had ASA scores of 3 and $10.6 \%$ of 4 . ASA scores and MET values, however, were not predictive for intraoperative blood loss, as they showed no correlation when correcting for multiple comparisons. When analyzing additional preoperative parameters, age did not show a correlation with intraoperative blood loss, as the deviation from zero of the linear regression was not significant $(\mathrm{p}=0.1925)$ (Fig. 3B). However, although no strong correlation $\left(r^{2}=0.037\right)$ could be observed, BMI positively correlated with intraoperative blood loss ( $p=0.0007)$ (Fig. 3C). In addition, surgery length correlated significantly with intraoperative blood loss $\left(r^{2}=0.1775, p<0.0001\right.$, linear regression). We also compared the pre- and intraoperative and first postoperative hemoglobin levels according to the patients who received 0, 1-3, and > 3 units of RBC transfusions (Fig. 3E). As expected, patients who did not need blood transfusions started with a higher preoperative hemoglobin level (mean $12.16 \mathrm{~g} / \mathrm{dl}$ ) compared to patients receiving 1-3 units or $>3$ units (mean preoperative hemoglobin levels 10.59 or 10.67 $\mathrm{mg} / \mathrm{dl}$, respectively). In all three groups, hemoglobin levels dropped significantly intraoperatively $(\mathrm{p}<0.0001$, oneway ANOVA). Hemoglobin levels in patients not requiring blood transfusion dropped on average by $1.89 \mathrm{mg} / \mathrm{dl}$, compared to $2.32 \mathrm{mg} / \mathrm{dl}$ and $2.64 \mathrm{mg} / \mathrm{dl}$ in patients receiving 1-3 units and $>3$ units of RBCs, respectively (Fig. 3E). Of note, in most patients not requiring blood transfusions, the hemoglobin level did not drop below $9 \mathrm{~g} / \mathrm{dl}$ (dashed line in Fig. 3E).

\section{Surgical Procedure}

Multivariate analysis primarily identified the type of anterior implant, i.e., cage versus Harms mesh versus ex-
TABLE 1. Patient population characteristics

\begin{tabular}{|c|c|}
\hline & Value \\
\hline \multicolumn{2}{|l|}{ Sex } \\
\hline Male & $269(62.6)$ \\
\hline Female & $161(37.4)$ \\
\hline Total & $430(100)$ \\
\hline Age, yrs, mean \pm SD (range) & $64.1 \pm 0.585(23-91)$ \\
\hline \multicolumn{2}{|l|}{$\mathrm{BMI}$} \\
\hline$<18.5$ & $26(6.2)$ \\
\hline $18.5-24.9$ & $206(48.8)$ \\
\hline $25.0-29.9$ & $135(32.0)$ \\
\hline $30.0-34.9$ & $39(9.2)$ \\
\hline$\geq 35.0$ & $16(3.8)$ \\
\hline \multicolumn{2}{|l|}{ ASA score } \\
\hline 1 & $3(0.7)$ \\
\hline 2 & $83(19.9)$ \\
\hline 3 & $293(70.1)$ \\
\hline 4 & $39(9.3)$ \\
\hline \multicolumn{2}{|l|}{ MET } \\
\hline$<4$ & $106(26.6)$ \\
\hline$\geq 4$ & $293(73.4)$ \\
\hline \multicolumn{2}{|l|}{ Metastasis histology } \\
\hline Breast cancer & $64(14.9)$ \\
\hline Colon cancer & $22(5.1)$ \\
\hline CUP & $17(4.0)$ \\
\hline Gastric cancer & $21(4.9)$ \\
\hline $\mathrm{HCC}$ & $11(2.6)$ \\
\hline Lung cancer & $65(15.1)$ \\
\hline Myeloma & $50(11.6)$ \\
\hline Prostate cancer & $69(16.0)$ \\
\hline Renal cancer & $36(8.4)$ \\
\hline Sarcoma & $15(3.5)$ \\
\hline Ureteral cancer & $10(2.3)$ \\
\hline Oropharygeal cancer & $18(4.2)$ \\
\hline Melanoma & $8(1.9)$ \\
\hline Other & $24(5.6)$ \\
\hline Total & $430(100.0)$ \\
\hline \multicolumn{2}{|l|}{ Metastasis location } \\
\hline Spine only & $206(48.0)$ \\
\hline Other organs & $223(52.0)$ \\
\hline Total & $429(100.0)$ \\
\hline
\end{tabular}

CUP = cancer of unknown primary origin; $\mathrm{HCC}=$ hepatocellular carcinoma. Values are presented as number (\%) of patients unless otherwise indicated.

pandable cage, and the stabilization technique, i.e., open versus percutaneous versus a combined dorsoventral procedure, as significant parameters associated with intraoperative blood loss. When stabilization and decompression only, in the sense of separation surgery, is not possible due to severe destruction of the vertebral body with loss of spinal alignment, resection of the affected vertebra(e) with Harms mesh or expandable cages as replacement is 


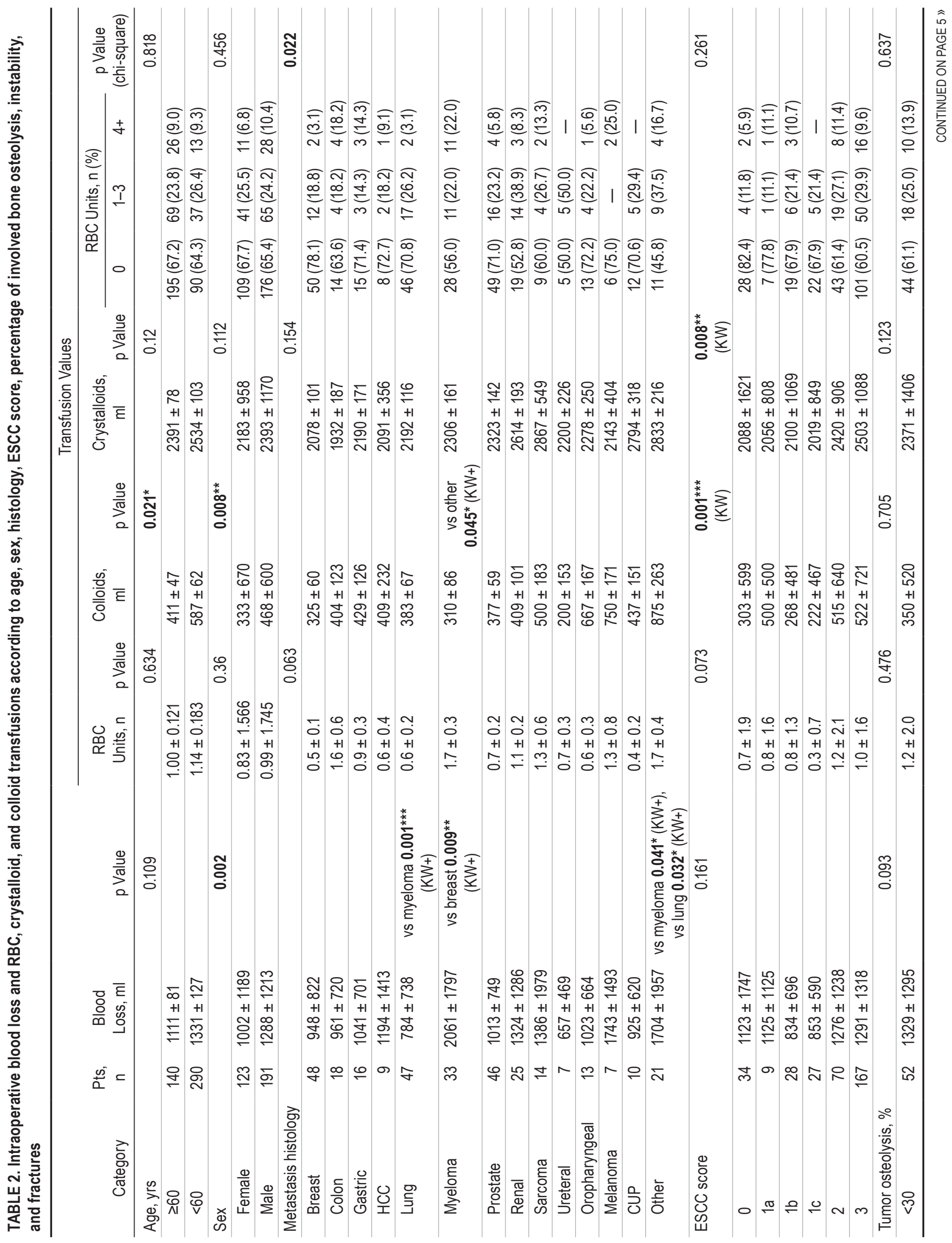




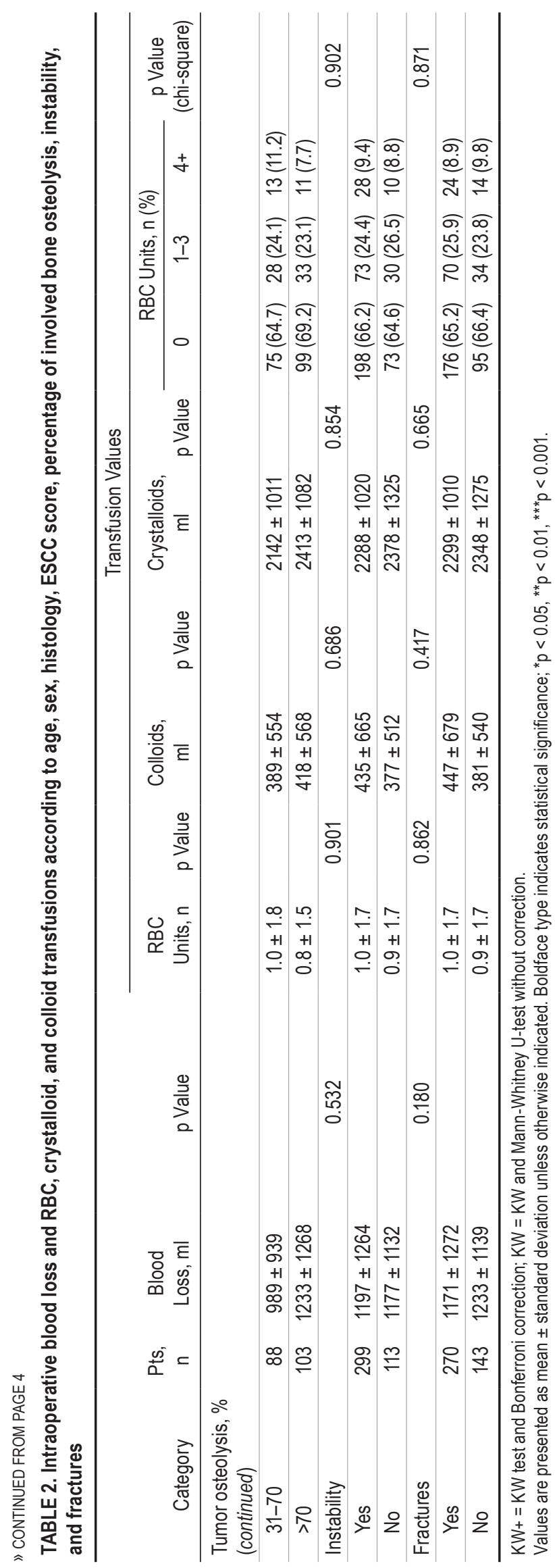

needed. Patients treated with Harms mesh and expandable cages required significantly more transfusions of at least one RBC blood product in $57.7 \%$ and $38.6 \%$ of cases, respectively, compared to solely dorsal procedures without vertebral body resection ( $\mathrm{p}=0.017$ ) (Fig. 4A). The decompression and stabilization strategy, as grouped by the approach (open, percutaneous, or combined), showed a significant association for RBC transfusions $(p=0.001)$ with open and combined procedures, whereas patients undergoing only percutaneous stabilization, as expected, rarely required transfusions. However, in patients undergoing a combined approach, RBC transfusions were needed in $41.7 \%$, versus $38.0 \%$ of patients with an open approach and $15.7 \%$ with a percutaneous approach (Fig. 4B).

\section{Multiple Linear Regression Analysis}

Based on our single-center analysis, we have summarized our findings in a preoperative checklist to identify patients at risk for receiving blood transfusions during oncological spine surgery. Compiling the five parameters with the greatest influence on intraoperative blood loss, the following parameters were included in the regression analysis: 1) histology including myeloma, renal cell carcinoma, or thyroid carcinoma, yes/no; 2) ESCC $\geq 2$, yes/no; 3) expected surgery length $>210$ minutes, yes/ no; 4) preoperative hemoglobin level $<11 \mathrm{mg} / \mathrm{dl}$, yes/no; and 5) surgical procedure including vertebral body resection (i.e., use of Harms mesh or expandable cage), yes/no. An initial regression model did not confirm the effect of preoperative high serum hemoglobin concentration $(>11$ $\mathrm{g} / \mathrm{L}$ ); therefore, this parameter was excluded from the final regression model. The final version included variables that significantly predicted a strong effect $(\mathrm{R}=0.437)$ of intraoperative blood loss ( $\mathrm{p}<0.001$ ) (Fig. 5). A blood loss increase of $621 \pm 152 \mathrm{ml}$ can be predicted when the primary tumor is renal cell carcinoma, thyroid carcinoma, or myeloma ( $\mathrm{p}<0.001)$. An ESCC $\geq 2$ resulted in an increase in blood loss of $239 \pm 125 \mathrm{ml}(\mathrm{p}=0.011)$. Increased blood losses also occurred with operative times longer than 210 minutes $(864 \pm 133 \mathrm{ml}, \mathrm{p}<0.001)$ and procedures involving implantation of a mesh or expandable cage $(341 \pm 155$ $\mathrm{ml}, \mathrm{p}=0.03)$. On the basis of these results, we added the parameter of serum hemoglobin $>11 \mathrm{~g} / \mathrm{L}$, which was not derived from the multiple linear regression analysis, to the clinical checklist as a surrogate for resilience concerning blood loss.

\section{Discussion}

Surgical planning in oncological spine surgery requires careful consideration of numerous factors impacting patient outcome. While the overall oncological strategy is being defined in interdisciplinary tumor boards, in this particularly vulnerable patient cohort the surgical risk assessment and operative strategy are usually defined by the spine surgeon. To improve preoperative risk assessment and transfusion management in conjunction with the anesthesiological partner, our study aimed to assess the risk factors for intraoperative blood loss in patients undergoing surgery for metastases of the spine.

The receipt of allogeneic blood transfusions (ABTs) is 


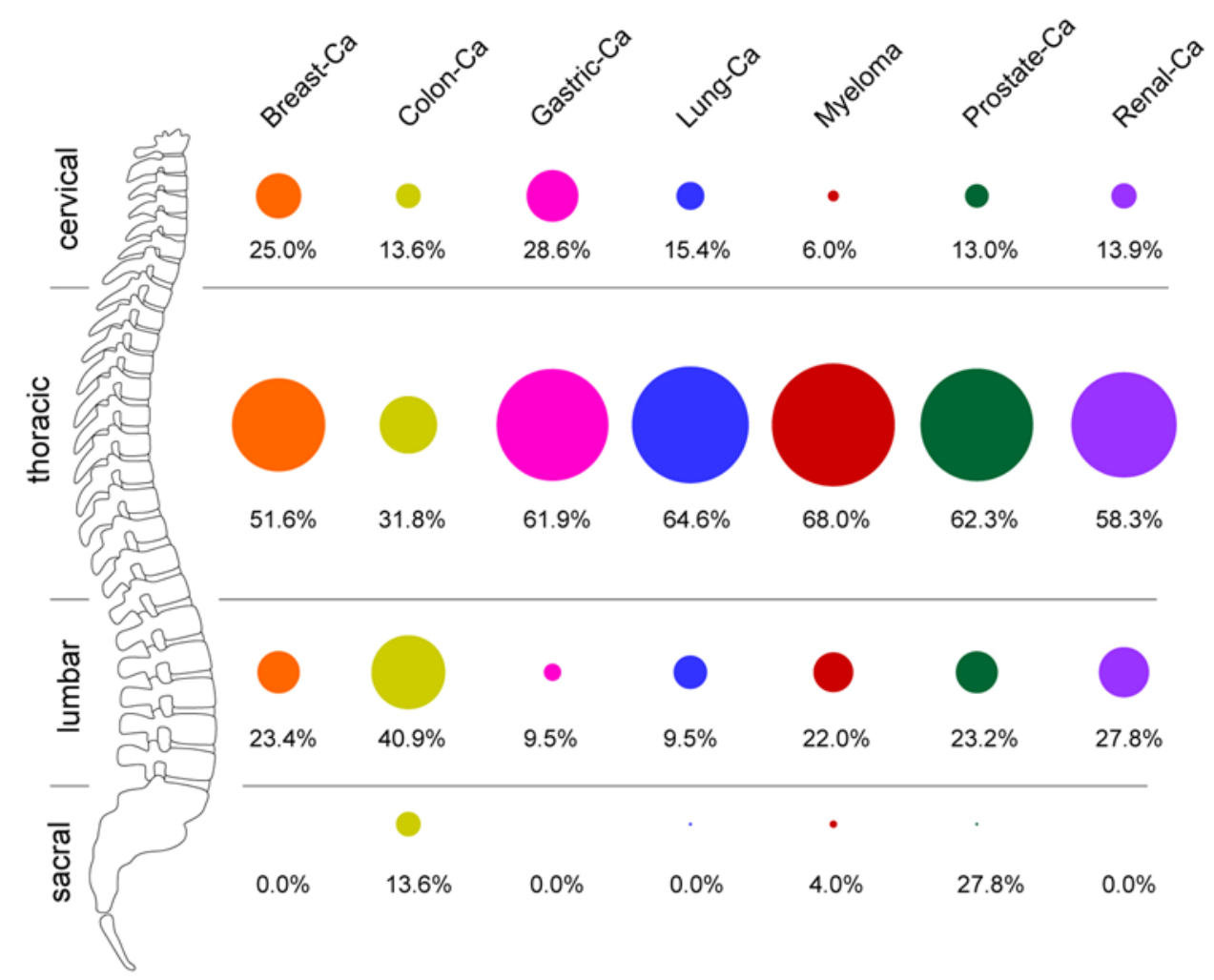

FIG. 1. Distribution of tumor histology for the most common entities (> 5\%) along the spinal axis. Dot size represents the distribution percentage; cervical, C0-T1; thoracic, T2-L1; lumbar, L2-S1; and sacral, S2-4. Ca = cancer.

associated with postoperative infections, ${ }^{15}$ thrombembolisms, ${ }^{16,17}$ and other adverse effects, even death. ${ }^{17}$ A retrospective analysis by Zaw et al. demonstrated that patients undergoing oncological spine surgery were at a twofold risk for overall perioperative complications when receiving ABTs..$^{18}$ Prospectively defining patients who are at risk for receiving ABTs can therefore help to enhance intraand perioperative management, including fluids, antibiotics, and coagulation, and improve the collaborative efforts of surgeons and anesthesiologists.

We found that among patients with spinal metastases, those with myeloma presented with a significantly increased intraoperative blood loss. Previous studies primarily identified renal and thyroid carcinoma as metastasis histologies associated with risks for increased intraoperative blood loss. ${ }^{19,20}$ In a systematic review of 18 smaller studies and case series by Chen et al., myeloma was repetitively found to be associated with increased intraoperative blood loss..$^{12}$ One explanation might be that in our cohort, patients with myeloma overall have a good oncological prognosis, and therefore a more aggressive surgical strategy was chosen. On the other hand, the pathophysiology of lymphoproliferative disorders is associated with alterations in blood coagulation. ${ }^{21}$ Although a hypercoagulative state is detected more frequently in these patients, an acquired von Willebrand syndrome can occur in patients with multiple myeloma. ${ }^{22}$ Hematological studies found that the paraprotein produced in multiple myeloma can have a significant effect on blood coagulation. ${ }^{21,23}$ For example, the ristocetin-induced platelet aggregation test, a measurement to determine the interaction of the platelet glycoprotein (GP) Ib $\alpha$-V-IX complex and von Willebrand factor (vWF), was reduced in $15 \%$ of all multiple myeloma patients, thus indicating impaired coagulation in the vWF axis. ${ }^{23}$

In the present study, the tumors in the myeloma patients proved to be highly vascularized intraoperatively. One approach for reducing the amount of intraoperative blood loss is preoperative embolization via the arterial flow, which is used in particular for hypervascularized metastases such as those in patients with renal cell carcinoma and thyroid cancer. Overall, 5 of 25 (20\%) renal cell carcinoma patients in our cohort underwent preoperative tumor embolization. This might explain why no significant association with increased intraoperative blood loss was found in our study, even if selected studies do not show any significant influence on intraoperative blood loss after tumor embolization. ${ }^{24-26}$ Nevertheless, an overall moderate positive effect of preoperative embolization can be assumed in hypervascularized tumor entities. ${ }^{27}$ In particular, systematic reviews from 2016 and 2018, respectively, by Griessenauer et al. and Luksanapruksa et al. confirm this suspected positive effect, but data from high-quality prospective randomized studies are lacking. ${ }^{28,29}$ It is also notable that additional studies in nonhypervascularized tumors showed a positive effect of embolization on intraoperative blood loss, indicating that more patients than previously suspected might benefit from this combined treatment. ${ }^{30-32}$ However, despite the proven positive effects of prior embolization on intraoperative blood loss, a complication rate of 

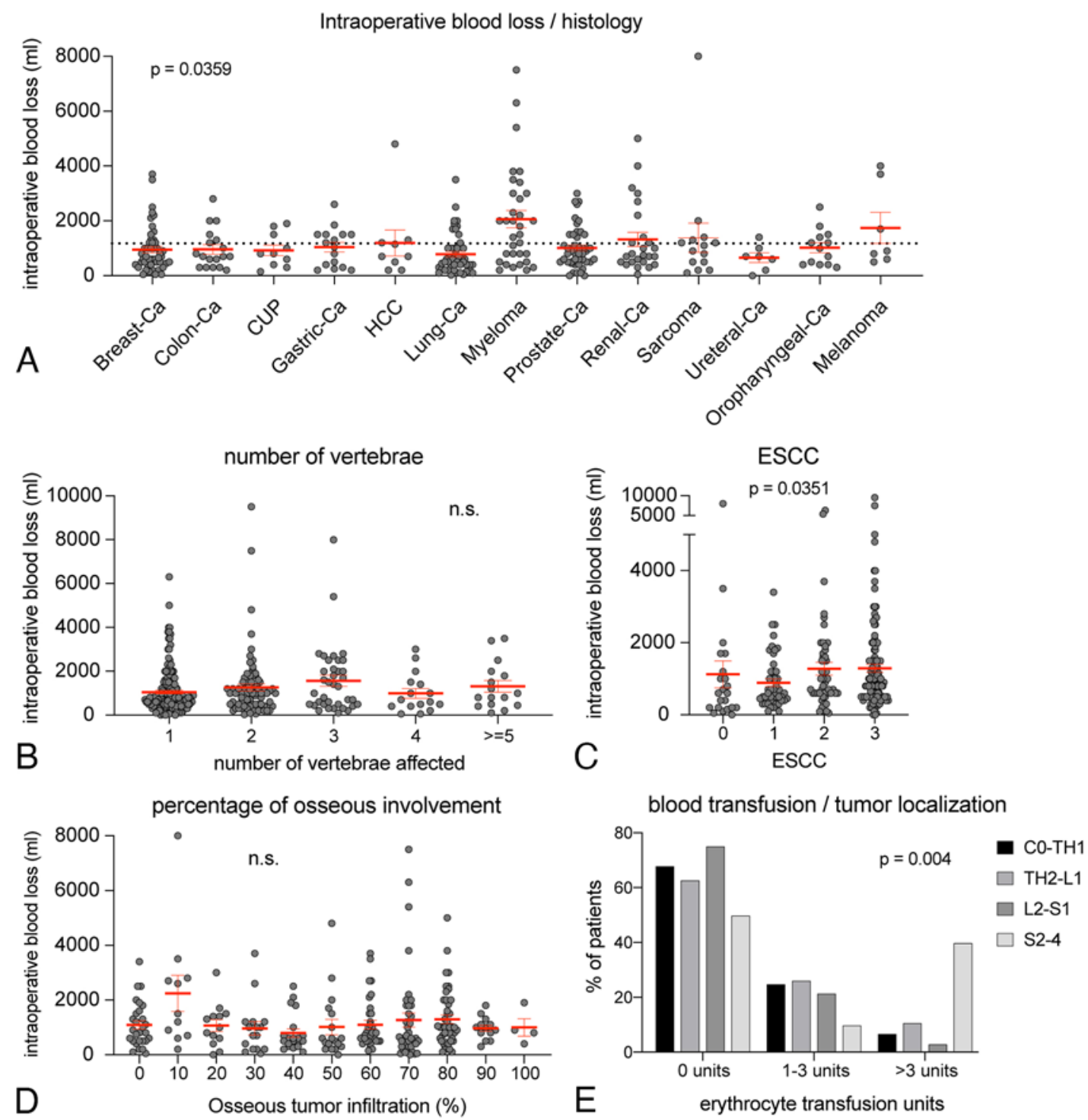

FIG. 2. Graphs depicting intraoperative blood loss according to tumor histology (A), number of vertebrae affected by the tumor (B), ESSC scale (C), and percentage of osseous infiltration of the tumor (D). Mean \pm SEM, KW test. Bar graph showing the percentage of patients requiring RBC transfusions according to tumor localization (chi-square test) (E). CUP = cancer of unknown primary origin; $\mathrm{HCC}=$ hepatocellular carcinoma.

around 3\% should also be included in the decision-making process. In this context, the decision to apply preoperative embolization has to be made on an individual basis until larger prospective studies have been conducted. . $^{2,28,29,33,34}$

In addition to known factors, such as the operative time and type of surgery, we investigated the usefulness of the routinely acquired preoperative ASA and MET scores. Although subjective, the ASA score is usually a good independent prognostic factor for perioperative morbidity. ${ }^{35}$ However, in our study, ASA and other anesthesiological scores had no prognostic association with intraoperative blood loss and the frequency of blood transfusion. This observation was confirmed in a study by Kumar et al., which did not find any association of these variables with the Charlson Comorbidity Index. ${ }^{19}$ In our opinion, only very few preoperative "anesthesiological" parameters, such as the hemoglobin level and associated baseline anemia, are useful to assess the risk for intraoperative RBC transfusions. Our view is supported by results reported by Pennington et al., who primarily found preoperative hematocrit, red cell distribution width, mean corpuscular volume, and preoperative albumin levels, as well as various surgical parameters, to be risk factors for intraoperative blood loss and the necessity for blood transfusions. ${ }^{36}$

Due to the increased overall perioperative risk associated with ABTs in spine surgery, as well as the intraoperative anesthesiological challenges in fluid and coagulation management, various strategies have aimed to decrease intraoperative blood loss in oncological spine surgery. ${ }^{37}$ The literature shows that preoperative embolization ${ }^{38,39}$ and use of antifibrinolytic agents, ${ }^{40,41}$ or even rare invasive approaches, such as aortic balloon occlusion, ${ }^{42,43}$ can be 


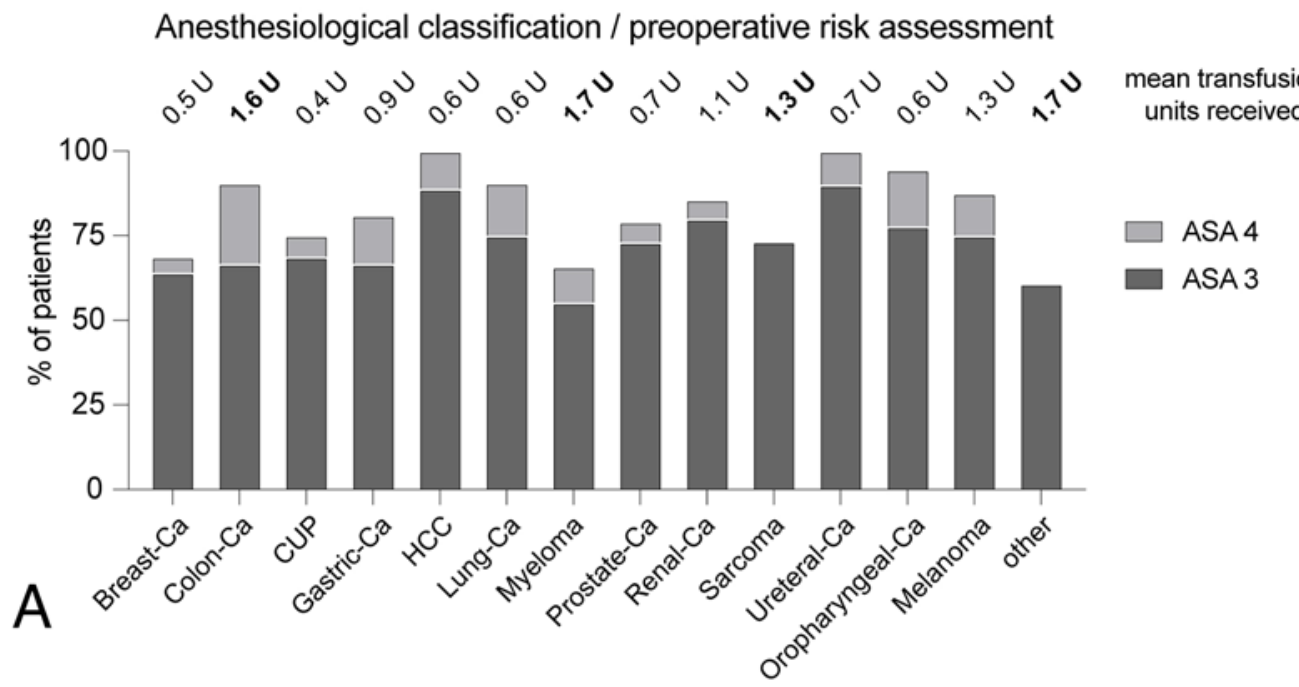

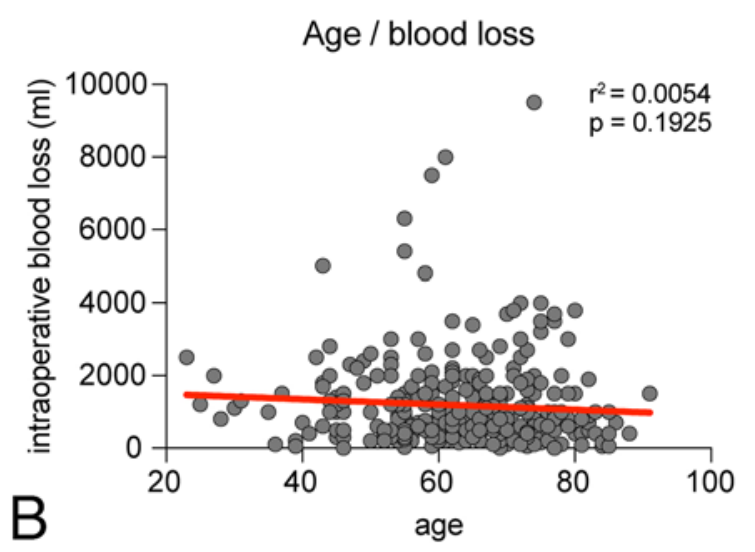

Surgery length / blood loss

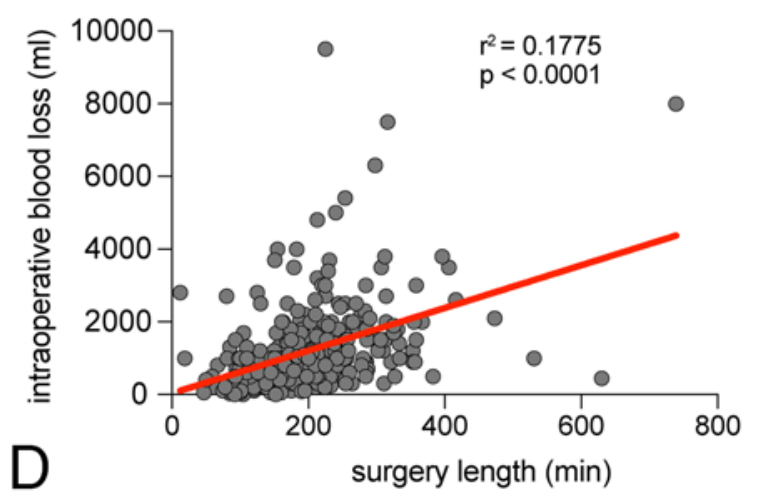

\section{BMl / blood loss}

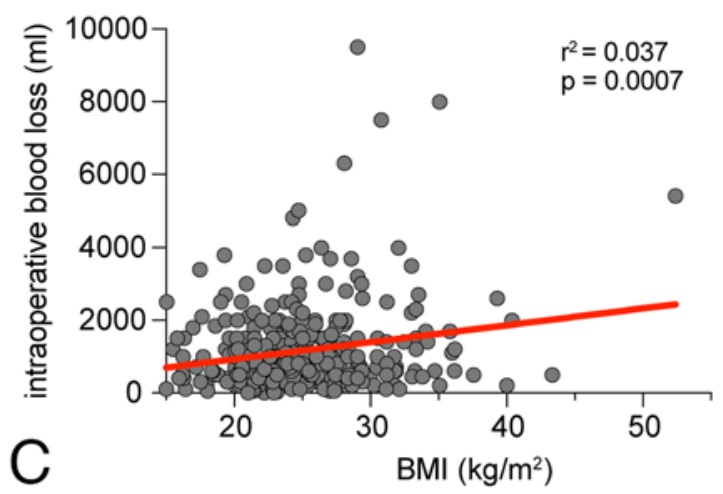

Hemoglobin dynamic intraoperatively

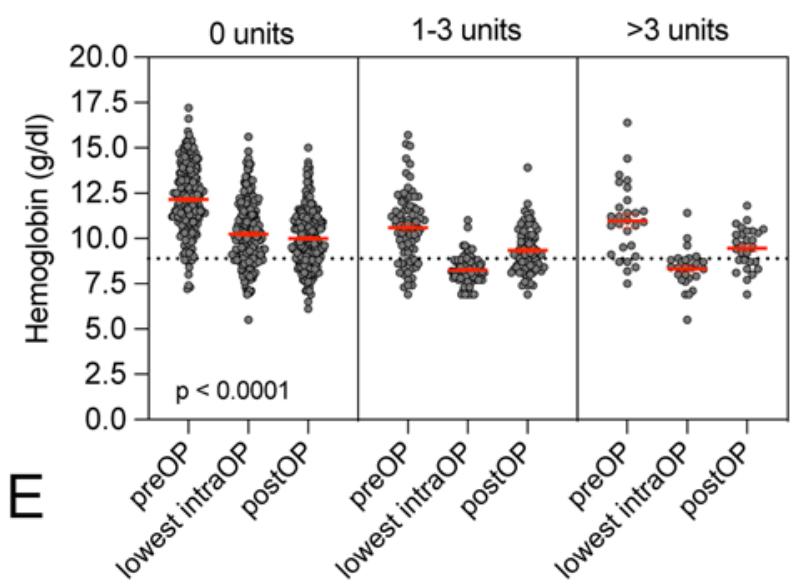

FIG. 3. Graph showing the percentage of patients with an ASA score of 3 (dark gray, severe disease) or 4 (light gray, severe incapacitating disease with constant threat to life), including the mean number of transfusion units by histology (A). Correlation analysis (simple linear regression analysis) of intraoperative blood loss ( $\mathrm{ml}$ ) association with age (B), BMI (C), and surgery length (minutes) (D). Hemoglobin level (g/dl) dynamic preoperatively (preOP), lowest intraoperatively (lowest intraOP), and postoperatively (postOP) stratified by the need for intraoperative transfusion of RBC units $(E)$. Mean \pm SEM, one-way ANOVA.

applied to decrease intraoperative blood loss. For adaptation of the surgical strategy by using minimally invasive or staged surgical approaches, embolization is the preoperative procedure that is most widely used at high-volume oncological spine centers, and a large retrospective review by Nair et al. demonstrated that preoperative embolization can be safely applied. ${ }^{39}$ Embolization in this cohort was primarily performed in patients with renal cell carci- 

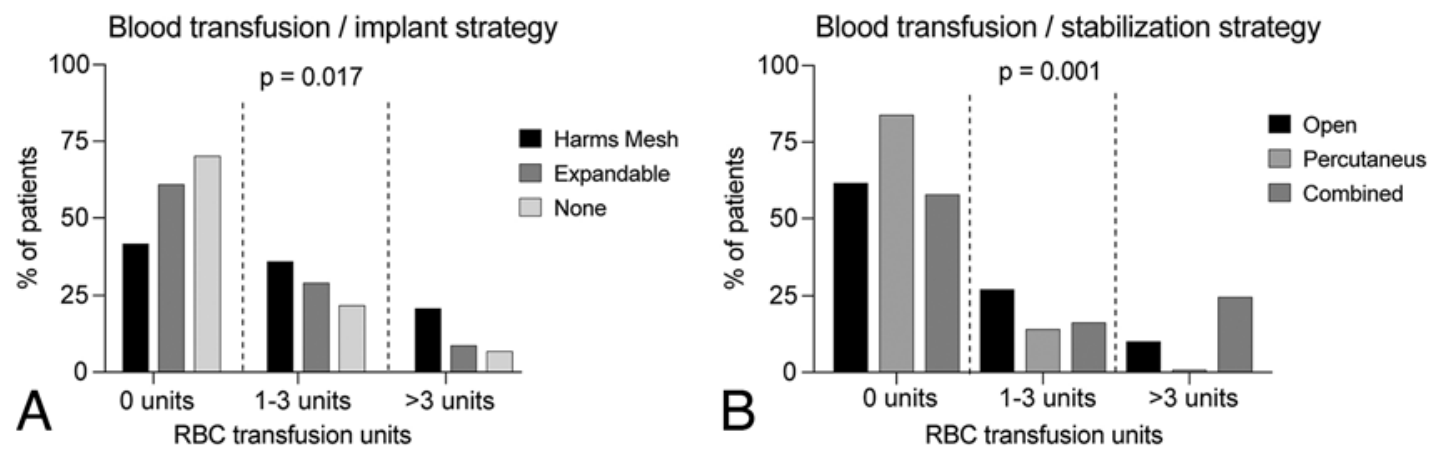

FIG. 4. A: Bar graph depicting the percentage of patients receiving different implant materials during tumor decompression (cage, Harms mesh, expandable cages, or no implants) stratified by number of RBC transfusion units received. Chi-square test. B: The same bar graph depicts the operative strategy, as defined by an open, percutaneous (e.g., percutaneous stabilization and biopsy), or combined stabilization approach. Chi-square test.

noma, thyroid carcinoma, and leiomyosarcoma. ${ }^{39}$ So far, to our knowledge, no study has investigated the effect of embolization on patients with myeloma, in whom tumors were demonstrated to be highly vascularized in our cohort. Besides embolization, selected orthopedic studies demonstrated that tranexamic acid, an antifibrinolytic agent, can be used effectively to reduce overall blood loss and transfusion requirements. ${ }^{37,44,45}$ As surgery transiently increased fibrinolysis and therefore increased the risk for major bleedings and intraoperative coagulation derangements in patients with hypervascularized tumors, administration of tranexamic acid at the beginning of surgery could be implemented in patients at risk for major bleeding complications, as identified by our checklist.

Because the use of ABTs, which still represent the gold standard for blood replenishment, may lead not only to an increased rate of postoperative infections, but also to stimulation of tumor growth through the consecutive immunosuppression, each administration should be critically examined and, if possible, avoided. ${ }^{46}$

The use of intraoperative cell salvage for autologous blood transfusion has not yet been sufficiently researched for spinal metastasis surgery, although evidence from surgery for gastrointestinal, gynecological, urological, and also lung cancer shows that negative effects in terms of tumor dissemination cannot be assumed when a leukocyte depletion filter is used. ${ }^{19,47}$ Autologous transfusion represents another option to reduce reliance on allogeneic blood products but is often precluded due to the significant preexisting diseases that are usually present as well as the urgency of the operation in patients with metastatic vertebral disease..$^{48}$

Previous studies analyzing intraoperative blood loss in spine surgery using retrospective databases have applied different approaches to generate a preoperative model that accurately predicts the intraoperative blood loos. The study by Gao et al..$^{20}$ performed correlative analysis of the tumor resection strategy and histology to identify major contributing factors for intraoperative blood loss before validating their prediction model on a test cohort. The correlation coefficient was 0.559 , including the tumor histology and a detailed description of the surgical approach. ${ }^{20} \mathrm{~A}$ more complex approach that included an online calculator was designed for use in a study by Pennington et al., who incorporated 12 parameters, including preoperative hematocrit, mean corpuscular hemoglobin, red cell distribution width, and albumin levels, to predict intraoperative blood loss. ${ }^{36}$ Their model achieved an optimism-corrected area under the curve of 0.819 . Both studies present potentially valuable tools for preoperative risk assessment in spine surgery. However, the "efficacy of simplicity," which can significantly improve the workflow in a fast-moving, interdisciplinary, and high-volume surgical field such as oncological spine surgery, should not be neglected. This is the reason why our aim was to generate a small but efficient checklist that can be used by surgeons and anesthesiologists in their daily routine to identify patients at risk for intraoperative blood loss. The checklist we present here can be implemented during the preoperative anesthesiological assessment and performed on a regular basis before spinal tumor surgery. Although the overall correlation coefficient of this short list does not compete with the more complex model by Pennington et al. ${ }^{36}$ we believe that the simplicity of our checklist will lead to high workflow compliance in routine practice.

The main limitation of our study is the single-center

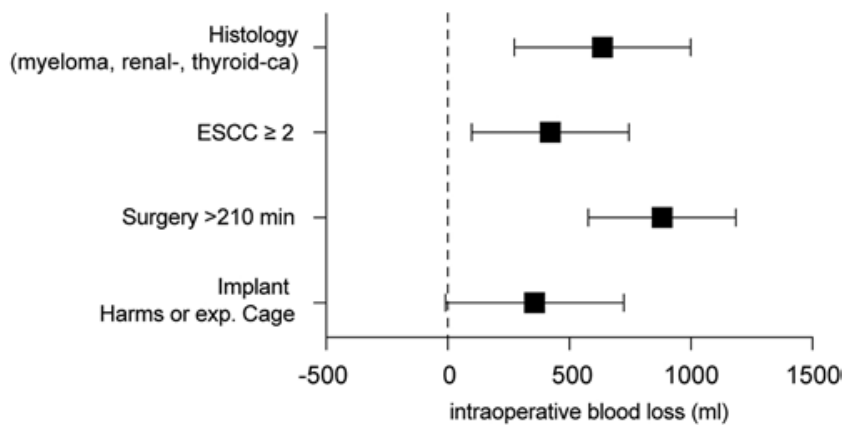

FIG. 5. Preoperative checklist recommendations based on a multiple linear regression analysis, showing the variables with the biggest impact on intraoperative blood loss: histology (including myeloma, renal carcinoma, and thyroid carcinoma), ESCC score, surgery duration > 210 minutes, and implant material (includes Harms cage and/or expandable [exp.] cage). Regression coefficient and $95 \% \mathrm{Cl}$. 
and retrospective study design. In addition, personal surgical skills have a decisive influence on intraoperative blood loss, although this aspect cannot be objectively analyzed. Furthermore, vertebral en bloc resections were not part of this analysis and should not be evaluated on the basis of the presented preoperative checklist due to the often very extensive procedure and the expected large amount of blood loss depending on the necessary further organ or vascular resections.

\section{Conclusions}

Taken together, our analysis represents to our knowledge the largest analysis of intra- and perioperative factors affecting intraoperative blood loss in oncological spine surgery so far. Our data show that myeloma presents intraoperatively with increased blood loss. In addition, while routine ASA scoring did not prove to be a good prognosticator for intraoperative transfusion requirements or blood loss, the ESCC score, BMI, and operative procedure length, as well as the type of implant, which is a surrogate for the surgical strategy, showed significant association with intraoperative blood loss. Based on our single-center analysis, we summarized our findings in a preoperative checklist to identify patients at risk for receiving blood transfusions during oncological spine surgery: 1) metastatic tumor histology including myeloma, renal cell carcinoma, or thyroid carcinoma; 2) ESCC $\geq 2$; 3) expected surgery length $>210$ minutes; 4) preoperative hemoglobin level $<11 \mathrm{~g} / \mathrm{L}$; and 5) surgical procedure involving implantation of a mesh or expandable cage. Surgeons and anesthesiologists can easily apply this checklist in their daily routine to identify patients at risk for intraoperative bleeding complications.

\section{References}

1. Guillevin R, Vallee JN, Lafitte F, et al. Spine metastasis imaging: review of the literature. J Neuroradiol. 2007;34(5): 311-321.

2. Sohn S, Kim J, Chung CK, et al. A nationwide epidemiological study of newly diagnosed spine metastasis in the adult Korean population. Spine J. 2016;16(8):937-945.

3. Laufer I, Rubin DG, Lis E, et al. The NOMS framework: approach to the treatment of spinal metastatic tumors. Oncologist. 2013;18(6):744-751.

4. Fisher CG, DiPaola CP, Ryken TC, et al. A novel classification system for spinal instability in neoplastic disease: an evidence-based approach and expert consensus from the Spine Oncology Study Group. Spine (Phila Pa 1976). 2010; 35(22):E1221-E1229.

5. Tomita K, Kawahara N, Kobayashi T, et al. Surgical strategy for spinal metastases. Spine (Phila Pa 1976). 2001;26(3): 298-306.

6. Tokuhashi Y, Matsuzaki $\mathrm{H}$, Oda $\mathrm{H}$, et al. A revised scoring system for preoperative evaluation of metastatic spine tumor prognosis. Spine (Phila Pa 1976). 2005;30(19):2186-2191.

7. Laufer I, Iorgulescu JB, Chapman T, et al. Local disease control for spinal metastases following "separation surgery" and adjuvant hypofractionated or high-dose single-fraction stereotactic radiosurgery: outcome analysis in 186 patients. $J$ Neurosurg Spine. 2013;18(3):207-214.

8. Moussazadeh N, Laufer I, Yamada Y, Bilsky MH. Separation surgery for spinal metastases: effect of spinal radiosurgery on surgical treatment goals. Cancer Contr. 2014;21(2):168-174.

9. Lu VM, Alvi MA, Goyal A, et al. The potential of minimally invasive surgery to treat metastatic spinal disease versus open surgery: a systematic review and meta-analysis. World Neurosurg. 2018;112:e859-e868.

10. Pennington Z, Ahmed AK, Molina CA, et al. Minimally invasive versus conventional spine surgery for vertebral metastases: a systematic review of the evidence. Ann Transl Med. 2018;6(6):103.

11. Barzilai O, Bilsky MH, Laufer I. The role of minimal access surgery in the treatment of spinal metastatic tumors. Global Spine J. 2020;10(2)(suppl):79S-87S.

12. Chen Y, Tai BC, Nayak D, et al. Blood loss in spinal tumour surgery and surgery for metastatic spinal disease: a metaanalysis. Bone Joint J. 2013;95-B(5):683-688.

13. Kumar N, Zaw AS, Khine HE, et al. Blood loss and transfusion requirements in metastatic spinal tumor surgery: evaluation of influencing factors. Ann Surg Oncol. 2016;23(6): 2079-2086.

14. Bilsky MH, Laufer I, Fourney DR, et al. Reliability analysis of the epidural spinal cord compression scale. J Neurosurg Spine. 2010;13(3):324-328.

15. He YK, Li HZ, Lu HD. Is blood transfusion associated with an increased risk of infection among spine surgery patients?: a meta-analysis. Medicine (Baltimore). 2019;98(28):e16287.

16. Aoude A, Nooh A, Fortin M, et al. Incidence, predictors, and postoperative complications of blood transfusion in thoracic and lumbar fusion surgery: an analysis of 13,695 patients from the American College of Surgeons National Surgical Quality Improvement Program Database. Global Spine J. 2016;6(8):756-764.

17. Aoude A, Aldebeyan S, Fortin M, et al. Prevalence and complications of postoperative transfusion for cervical fusion procedures in spine surgery: an analysis of 11,588 patients from the American College of Surgeons National Surgical Quality Improvement Program Database. Asian Spine J. 2017;11(6):880-891.

18. Zaw AS, Kantharajanna SB, Maharajan K, et al. Metastatic spine tumor surgery: does perioperative blood transfusion influence postoperative complications? Transfusion. 2017; 57(11):2790-2798.

19. Kumar N, Zaw AS, Khoo BL, et al. Intraoperative cell salvage in metastatic spine tumour surgery reduces potential for reinfusion of viable cancer cells. Eur Spine J. 2016;25(12): 4008-4015.

20. Gao X, Fan T, He S, et al. A useful model for predicting intraoperative blood loss in metastatic spine tumor surgery. Clin Spine Surg. 2020;33(6):E256-E262.

21. Coppola A, Tufano A, Di Capua M, Franchini M. Bleeding and thrombosis in multiple myeloma and related plasma cell disorders. Semin Thromb Hemost. 2011;37(8):929-945.

22. Jakway JL. Acquired von Willebrand's disease. Hematol Oncol Clin North Am. 1992;6(6):1409-1419.

23. Djunic I, Elezovic I, Ilic V, et al. Acquired von Willebrand syndrome in multiple myeloma. Hematology. 2011;16(4):209-212.

24. Thiex R, Harris MB, Sides C, et al. The role of preoperative transarterial embolization in spinal tumors. A large singlecenter experience. Spine J. 2013;13(2):141-149.

25. Clausen C, Dahl B, Frevert SC, et al. Preoperative embolization in surgical treatment of spinal metastases: single-blind, randomized controlled clinical trial of efficacy in decreasing intraoperative blood loss. J Vasc Interv Radiol. 2015;26(3): 402-12.e1.

26. Reitz M, Mende KC, Cramer C, et al. Surgical treatment of spinal metastases from renal cell carcinoma-effects of preoperative embolization on intraoperative blood loss. Neurosurg Rev. 2018;41(3):861-867.

27. Wilson MA, Cooke DL, Ghodke B, Mirza SK. Retrospective analysis of preoperative embolization of spinal tumors. AJNR Am J Neuroradiol. 2010;31(4):656-660.

28. Luksanapruksa P, Buchowski JM, Tongsai S, et al. Systematic 
review and meta-analysis of effectiveness of preoperative embolization in surgery for metastatic spine disease. $\mathrm{J} \mathrm{Neu}$ rointerv Surg. 2018;10(6):596-601.

29. Griessenauer CJ, Salem M, Hendrix P, et al. Preoperative embolization of spinal tumors: a systematic review and metaanalysis. World Neurosurg. 2016;87:362-371.

30. Yoo SL, Kim YH, Park HY, et al. Clinical significance of preoperative embolization for non-hypervascular metastatic spine tumors. J Korean Neurosurg Soc. 2019;62(1):106-113.

31. Hong CG, Cho JH, Suh DC, et al. Preoperative embolization in patients with metastatic spinal cord compression: mandatory or optional? World J Surg Oncol. 2017;15(1):45.

32. Kato S, Murakami H, Minami T, et al. Preoperative embolization significantly decreases intraoperative blood loss during palliative surgery for spinal metastasis. Orthopedics. 2012;35(9):e1389-e1395.

33. Kato S, Hozumi T, Takaki Y, et al. Optimal schedule of preoperative embolization for spinal metastasis surgery. Spine (Phila Pa 1976). 2013;38(22):1964-1969.

34. Houten JK, Swiggett SJ, Hadid B, et al. Neurologic complications of preoperative embolization of spinal metastasis: a systemic review of the literature identifying distinct mechanisms of injury. World Neurosurg. 2020;143:374-388.

35. Daabiss M. American Society of Anaesthesiologists physical status classification. Indian J Anaesth. 2011;55(2):111-115.

36. Pennington Z, Ehresman J, Feghali J, et al. A clinical calculator for predicting intraoperative blood loss and transfusion risk in spine tumor patients. Spine J. 2021;21(2):302-311.

37. Mikhail C, Pennington Z, Arnold PM, et al. Minimizing blood loss in spine surgery. Global Spine J. 2020;10(1)(suppl):71S-83S

38. Westbroek EM, Pennington Z, Ahmed AK, et al. Comparison of complete and near-complete endovascular embolization of hypervascular spine tumors with partial embolization. $J$ Neurosurg Spine. 2020;33(2):245-251.

39. Nair S, Gobin YP, Leng LZ, et al. Preoperative embolization of hypervascular thoracic, lumbar, and sacral spinal column tumors: technique and outcomes from a single center. Interv Neuroradiol. 2013;19(3):377-385.

40. Damade C, Tesson G, Gilard V, et al. Blood loss and perioperative transfusions related to surgery for spinal tumors. Relevance of tranexamic acid. Neurochirurgie. 2019;65(6): 377-381.

41. Neilipovitz DT. Tranexamic acid for major spinal surgery. Eur Spine J. 2004;13(suppl 1):S62-S65.

42. Zhang Y, Guo W, Tang X, et al. Can aortic balloon occlusion reduce blood loss during resection of sacral tumors that extend into the lower lumber spine? Clin Orthop Relat Res. 2018;476(3):490-498.
43. Tang X, Guo W, Yang R, et al. Use of aortic balloon occlusion to decrease blood loss during sacral tumor resection. $J$ Bone Joint Surg Am. 2010;92(8):1747-1753.

44. Farrow LS, Smith TO, Ashcroft GP, Myint PK. A systematic review of tranexamic acid in hip fracture surgery. $\mathrm{Br} J \mathrm{Clin}$ Pharmacol. 2016;82(6):1458-1470.

45. Yu CC, Gao WJ, Yang JS, et al. Can tranexamic acid reduce blood loss in cervical laminectomy with lateral mass screw fixation and bone grafting: a retrospective observational study. Medicine (Baltimore). 2017;96(5):e6043.

46. Blajchman MA, Bordin JO. The tumor growth-promoting effect of allogeneic blood transfusions. Immunol Invest. 1995; 24(1-2):311-317.

47. Kumar N, Chen Y, Zaw AS, et al. Use of intraoperative cellsalvage for autologous blood transfusions in metastatic spine tumour surgery: a systematic review. Lancet Oncol. 2014; 15(1):e33-e41.

48. Brookfield KF, Brown MD, Henriques SM, et al. Allogeneic transfusion after predonation of blood for elective spine surgery. Clin Orthop Relat Res. 2008;466(8):1949-1953.

\section{Disclosures}

Dr. Dreimann is supported by Stryker, Medtronic, Spineart (speaker), Medtronic (research support), and Spineart (advisory board).

\section{Author Contributions}

Conception and design: Mohme, Dreimann, Krätzig, Stangenberg. Acquisition of data: Mohme, Pantel, Krätzig, Stangenberg. Analysis and interpretation of data: Mohme, Mende, Viezens, Eicker, Krätzig, Stangenberg. Drafting the article: Mohme, Mende, Eicker, Krätzig, Stangenberg. Critically revising the article: Mohme, Pantel, Viezens, Eicker, Dreimann, Krätzig, Stangenberg. Reviewed submitted version of manuscript: Mohme, Pantel, Viezens, Dreimann, Krätzig, Stangenberg. Approved the final version of the manuscript on behalf of all authors: Mohme. Statistical analysis: Mende. Administrative/technical/material support: Mohme, Westphal, Eicker, Krätzig, Stangenberg. Study supervision: Mohme, Viezens, Westphal, Eicker, Dreimann, Krätzig, Stangenberg.

\section{Correspondence}

Malte Mohme: University Medical Center Hamburg-Eppendorf, Hamburg, Germany.m.mohme@uke.de. 